\title{
Void growth to coalescence in a non-local material
}

\section{Niordson, Christian Frithiof}

\section{Published in:}

European Journal of Mechanics A - Solids

Link to article, DOI:

10.1016/j.euromechsol.2007.07.001

Publication date:

2008

Link back to DTU Orbit

Citation (APA):

Niordson, C. F. (2008). Void growth to coalescence in a non-local material. European Journal of Mechanics $A$ Solids, 27(2), 222-233. https://doi.org/10.1016/j.euromechsol.2007.07.001

\section{General rights}

Copyright and moral rights for the publications made accessible in the public portal are retained by the authors and/or other copyright owners and it is a condition of accessing publications that users recognise and abide by the legal requirements associated with these rights.

- Users may download and print one copy of any publication from the public portal for the purpose of private study or research.

- You may not further distribute the material or use it for any profit-making activity or commercial gain

- You may freely distribute the URL identifying the publication in the public portal

If you believe that this document breaches copyright please contact us providing details, and we will remove access to the work immediately and investigate your claim. 


\title{
Void growth to coalescence in a non-local material
}

\author{
Christian F. Niordson \\ Department of Mechanical Engineering, Solid Mechanics \\ Technical University of Denmark \\ DK-2800 Kgs. Lyngby, Denmark
}

July 23, 2008

Keywords : Void growth; Coalescence; Non-local plasticity; Size effects

\begin{abstract}
The size-effect in metals containing distributed spherical voids is analyzed numerically using a finite strain generalization of a length scale dependent plasticity theory. Results are obtained for stress-triaxialities relevant in front of a crack tip in an elastic-plastic metal. The influence of different material length parameters in a multi-parameter theory is studied, and it is shown that the important length parameter is the same as under purely hydrostatic loading. It is quantified how micron scale voids grow less rapidly than larger voids, and the implications of this in the overall strength of the material is emphasized. The size effect on the onset of coalescence is studied, and results for the void volume fraction and the strain at the onset of coalescence are presented. It is concluded that for cracked specimens not only the void volume fraction, but also the typical void size is of importance to the fracture strength of ductile materials.
\end{abstract}

\section{INTRODUCTION}

The resistance to crack growth in ductile materials depends strongly on nucleation and growth of voids, which span several orders of magnitude from the sub-micron range to about 100 microns. Voids can develop from micro-cracks nucleated at second phase particles by decohesion or by particle fracture, and the characterization of the growth of such voids under different loading conditions is important in order to understand ductile crack growth quantitatively. Extensive 
research has been directed toward modeling void growth and coalescence in order to obtain a quantitative understanding of the influence of void distribution, material properties and loading, on the basic mechanisms of void growth, and on bulk properties such as material response and fracture toughness (see e.g. Rice and Tracey, 1969; Needleman, 1972; Gurson, 1977; Tvergaard, 1982; Koplik and Needleman, 1988; Kysar et al., 2005; McElwain et al., 2006). Studies focussing on the effect of void shape have also been carried out by Gologanu et al. (1993, 1994) and Pardoen and Hutchinson (2000). These studies all rest on conventional models of plasticity, which is size-independent. The focus of the present paper is on quantifying the size-effect in void growth and coalescence relevant for sub-micron to micron size voids.

Due to gradient hardening metals exhibit size-effects on the micron scale. This has been experimentally confirmed in a number of investigations such as in indentation (Stelmashenko et al., 1993; Ma and Clarke, 1995; Swadener et al., 2002), in torsion (Fleck et al., 1994), and in bending (Stölken and Evans, 1998; Haque and Saif, 2003). A number of models have been proposed to model gradient hardening in metals. Among these are the lower-order theories by Acharya and Bassani (1996) and Huang et al. (2004). However, most of the models that have been proposed are of higher order nature resting on higher order stresses as work-conjugates to higher order strains, and using higher order boundary conditions (Aifantis, 1984; Fleck and Hutchinson, 1997, 2001; Gao et al., 1999; Gurtin, 2002; Gudmundson, 2004).

The experimental evidence of size-effects on the micron scale in metal plasticity, implies that growth of micron sized voids also exhibit important size-effects. This can also be concluded based on the close relationship that exists for the deformation fields under indentation and void growth (Marsh, 1964; Johnson, 1970). Since size-effects in indentation are widely observed in experiments (Stelmashenko et al., 1993; Ma and Clarke, 1995; Swadener et al., 2002), size effects must also be important in void growth as argued by Wei and Hutchinson (2003). Several researchers have investigated size-dependent void growth of single voids under hydrostatic loading (Fleck and Hutchinson, 1997; Huang et al., 2000; Fleck and Hutchinson, 2001). Liu et al., 2003 have extended the Rice-Tracey model of spherical symmetric void growth to account size-effects, while Liu et al., 2005 have extended this model to account for changes in the void shape during growth. A modified Gurson model accounting for the void size effect was proposed by Wen et al. (2005). Niordson and Tvergaard $(2006,2007)$ have studied the influence of constitutive length parameters on cavitation instabilities under very high stress triaxialities, 
for spherical and cylindrical voids. These void studies all aim at quantifying the intrinsic size effect in porous materials under macroscopically homogeneous loading, due to strain gradients on the micron scale around the voids. The main conclusion from the above studies is that void growth is suppressed on the micron scale, which leads to increased strength and ductility of the material, and that cavitation instabilities are delayed significantly.

A different form of size-effect in porous materials can be modeled using the nonlocal version of the Gurson model developed by Leblond et al. (1994). In this model a delocalization effect is introduced through a nonlocal measure of the porosity, and the associated material length parameter pertains to the size of the voids and their distribution. Using cell model analyses of localization in a void-sheet Tvergaard and Needleman (1997) found that the material length parameter in this nonlocal model scales with the void radius when shear bands develop, whereas it scales with the void spacing when localization takes place in a void-sheet perpendicular to the major principal loading direction.

In the present paper the intrinsic size-effect in porous materials, due to void size, is studied under moderate stress triaxialities as those in front of a crack tip in an elastic-plastic material. The interaction of neighboring voids is accounted for using an axi-symmetric cell model, which enables modeling of void growth to coalescence in a material with a uniform distribution of initially spherical voids. The size-effect modeled here pertains to gradient hardening on the micron scale.

\section{MATERIAL MODEL}

In order to model size-effects in void growth, the material around the voids is described by length-scale dependent finite strain plasticity theory. The theory which is used is that of Fleck and Hutchinson (2001), which was generalized to finite strains in Niordson and Redanz (2004).

The strain gradient plasticity theory by Fleck and Hutchinson (2001) employs three quadratic invariants of the gradient of the plastic strain rate $\rho_{i j k}=\rho_{j i k}=\dot{\epsilon}_{i j, k}^{P}$ in order to introduce hardening due to strain gradients. Plastic work in the material is performed due to the effective plastic strain measure, $E^{P}$, defined by the incremental relation

$$
\dot{E}^{P^{2}}=\dot{\epsilon}^{P^{2}}+l_{1}^{2} I_{1}+4 l_{2}^{2} I_{2}+\frac{8}{3} l_{3}^{2} I_{3}
$$

Here, $\dot{\epsilon}^{P^{2}}=\frac{2}{3} \dot{\epsilon}_{i j}^{P} \dot{\epsilon}_{i j}^{P}$ is the conventional measure of effective plastic strain rate, $I_{1}, I_{2}$ and $I_{3}$ are the three invariants of $\rho_{i j k}$, and $l_{1}, l_{2}$ and $l_{3}$ are three material length parameters, defining the 
length-scale on which gradient hardening becomes important.

A single parameter model, closely related to the theory by Aifantis (1984), was also proposed by Fleck and Hutchinson (2001). For this model the effective plastic strain is defined by

$$
\dot{E}^{P^{2}}=\dot{\epsilon}^{P^{2}}+l_{*}^{2} \dot{\epsilon}_{, i}^{P} \dot{\epsilon}_{, i}^{P}
$$

where $l_{*}$ is an alternative material length parameter.

The plastic strain increment is defined according to the usual relation for $J_{2}$ flow theory

$$
\dot{\epsilon}_{i j}^{P}=\frac{3}{2} \frac{S_{i j}}{\sigma_{(e)}} \dot{\epsilon}^{P}=m_{i j} \dot{\epsilon}^{P}
$$

where $S_{i j}$ are the Cartesian components of the deviator of the Cauchy stress $\sigma_{i j}$, the von Mises' effective stress is $\sigma_{(e)}=\sqrt{\frac{3}{2} S_{i j} S_{i j}}$, and $m_{i j}$ denotes the direction of the stress deviator.

Rewriting Equation (1) in terms of $m_{i j}$ and $\dot{\epsilon}^{P}$ results in

$$
\dot{E}^{P^{2}}=\dot{\epsilon}^{P^{2}}+A_{i j} \dot{\epsilon}_{, i}^{P} \dot{\epsilon}_{, j}^{P}+B_{i} \dot{\epsilon}_{, i}^{P} \dot{\epsilon}^{P}+C \dot{\epsilon}^{P^{2}}
$$

where the tensors $A_{i j}, B_{i}$ and $C$ depend on the three material length parameters $l_{1}, l_{2}$ and $l_{3}$ as well as on the spatial gradients of $m_{i j}$ (for details see Fleck and Hutchinson, 2001).

Aiming at an updated Lagrangian formulation the principle of virtual work is stated using the current configuration as reference (see Niordson and Redanz, 2004)

$$
\begin{aligned}
& \int_{V}\left(\stackrel{\nabla}{\varsigma}_{i j} \delta \dot{\epsilon}_{i j}-\sigma_{i j}\left(2 \dot{\epsilon}_{i k} \delta \dot{\epsilon}_{k j}-\dot{e}_{k j} \delta \dot{e}_{k i}\right)+\left(\dot{q}-\dot{\sigma}_{(e)}^{\varsigma}\right) \delta \dot{\epsilon}^{P}+\stackrel{\vee}{\rho}_{i} \delta \dot{\epsilon}_{, i}^{P}\right) d V \\
& =\int_{S}\left(\dot{T}_{0 i} \delta \dot{u}_{i}+\dot{t}_{0} \delta \dot{\epsilon}^{P}\right) d S
\end{aligned}
$$

The Jaumann rate of the Kirchhoff stress is denoted $\stackrel{\nabla}{\varsigma}_{i j}$, while $\dot{q}$ is the work conjugate to the plastic strain, $\epsilon^{P}$, and $\stackrel{\vee}{\rho}_{i}$ is the convected derivative of the higher order Kirchhoff stress. With the displacement vector denoted by $u_{i}$, the total strain rate is denoted by $\dot{\epsilon}_{i j}=\frac{1}{2}\left(\dot{u}_{i, j}+\dot{u}_{j, i}\right)$, and the rate of the displacement gradient is $\dot{e}_{i j}=\dot{u}_{i, j}$. The determinant of the metric tensor is denoted $J$, and the relation between the Kirchhoff stress, $\varsigma_{i j}$, and the Cauchy stress, $\sigma_{i j}$, is given by $\varsigma_{i j}=J \sigma_{i j}$. Similarly, the relation between the higher order Kirchhoff stress, $\rho_{i}$, and the true higher order stress, $\tau_{i}$, is given by $\rho_{i}=J \tau_{i}$. The effective stress enters the principle of virtual work through $\sigma_{(e)}^{\varsigma}=J \sigma_{(e)}$. The right-hand side of equation (5) consists of the regular traction displacement term in addition to a higher order traction term. Hence, higher order boundary 
conditions need to be specified in addition to conventional traction displacement boundary conditions. The nominal tractions and higher order tractions are denoted $\dot{T}_{0 i}$ and $\dot{t}_{0}$, respectively. For the present problem $\dot{t}_{0}=0$ is imposed as the higher order boundary conditions in all cases, on internal elastic-plastic boundaries as well as external boundaries, as was done in (Niordson and Tvergaard 2007) for plane strain void growth and in (Niordson and Tvergaard 2006) for the analyses of cavitation instabilities.

The constitutive equations for the Kirchhoff stress-measures are (see Niordson and Redanz, 2004)

$$
\begin{aligned}
\stackrel{\nabla}{\varsigma}_{i j} & =\mathcal{R}_{i j k l}\left(\dot{\epsilon}_{k l}-\dot{\epsilon}^{P} m_{k l}\right)=\dot{\varsigma}_{i j}-\dot{\omega}_{i k} \sigma_{k j}-\sigma_{i k} \dot{\omega}_{j k} \\
\dot{q} & =h\left(\dot{\epsilon}^{P}+\frac{1}{2} B_{i} \dot{\epsilon}_{, i}^{P}+C \dot{\epsilon}^{P}\right) \\
\vee_{\rho_{i}} & =h\left(A_{i j} \dot{\epsilon}_{, j}^{P}+\frac{1}{2} B_{i} \dot{\epsilon}^{P}\right)=\dot{\rho}_{i}-\dot{e}_{i k} \rho_{k}
\end{aligned}
$$

where $h=h\left[E^{p}\right]$ is the hardening modulus, and $\dot{\omega}_{i j}$ is the anti-symmetric part of $\dot{e}_{i j}$. The elastic stiffness tensor is given by

$$
\mathcal{R}_{i j k l}=\frac{E}{1+\nu}\left(\frac{1}{2}\left(\delta_{i k} \delta_{j l}+\delta_{i l} \delta_{j k}\right)+\frac{\nu}{1-2 \nu} \delta_{i j} \delta_{k l}\right)
$$

where $E$ is Young's modulus, $\nu$ is Poisson's ratio, and $\delta_{i j}$ is Kronecker's delta. The hardening modulus is calculated on the basis of the tangent-modulus through

$$
h\left[E_{T}\right]=\left(\frac{1}{E_{T}}-\frac{1}{E}\right)^{-1}
$$

The material surrounding the voids is assumed to be power-law hardening, using the relation between the effective plastic strain and the tangent modulus of the uniaxial stress-strain curve:

$$
E_{T}=\frac{E}{n}\left(\frac{E^{P}}{\epsilon_{0}}+1\right)^{(1 / n)-1}
$$

where $n$ is the hardening exponent and $\epsilon_{0}=\sigma_{y} / E$ is the yield strain under uni-axial tension, with $\sigma_{y}$ denoting the initial yield stress.

\section{NUMERICAL METHOD AND PROBLEM DESCRIPTION}

Numerical solutions are obtained using a two-field finite element method similar to that used by de Borst and Mühlhaus (1992), and de Borst and Pamin (1996). This method has also been used 


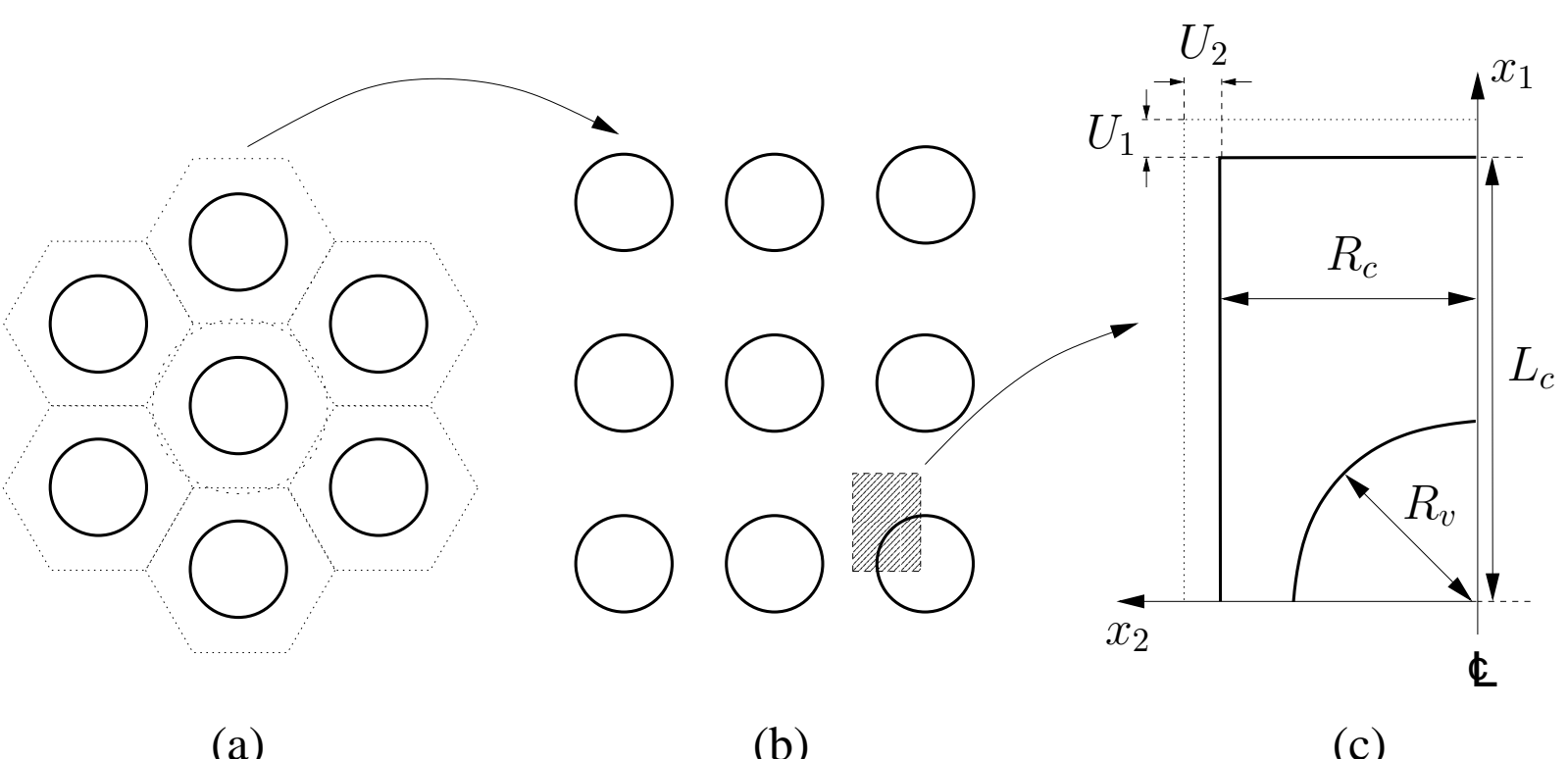

(a)

(b)

(c)

Figure 1: Cell model for a material with an array of voids. (a) Hexagonal distribution of voids with a cylindrical cell indicated by the circular dashed line. (b) A part of a layer of voids. (c) Using the symmetry of the problem half a void can be modeled in an axi-symmetric cell. The cell radius and length are denoted $R_{c}$ and $L_{c}$, respectively, and the void radius is denoted $R_{v}$.

by Niordson and Hutchinson (2003) to model the small strain theory by Fleck and Hutchinson (2001), while Niordson and Redanz (2004) and Niordson and Tvergaard (2005, 2006, 2007) have used the approach to solve problems at finite strains.

An axi-symmetric cell model is used to model a hexagonal arrangement of spherical voids (Figure 1a). The voids are aligned in planes as shown in Figure 1b. Using the symmetry of the problem the material can be analyzed numerically using the computational cell shown in Figure 1c. The void and cell radii are denoted $R_{v}$, and $R_{c}$, respectively, and the length of the cell is denoted $L_{c}$. Hence the in-plane void spacing is $2 R_{c}$, while the out-of-plane void spacing is $2 L_{c}$.

The void volume fraction for the material can be expressed in terms of the cell and void dimensions by

$$
f=\frac{2 R_{v}^{3}}{3 R_{c}^{2} L_{c}}
$$

For the results presented throughout this paper $L_{c} / R_{c}=1$ is used so that the in-plane and 
out-of-plane spacings are equal. The boundary conditions applied to the cell are specified by

$$
\begin{aligned}
& \dot{u}_{1}=0 \quad \text { and } \dot{T}_{02}=0, \quad \text { for } \quad x_{1}=0 \\
& \dot{u}_{1}=\dot{U}_{1} \text { and } \dot{T}_{02}=0, \quad \text { for } \quad x_{1}=L_{c}+U_{1} \\
& \dot{u}_{2}=0 \quad \text { and } \dot{T}_{01}=0, \quad \text { for } \quad x_{2}=0 \\
& \dot{u}_{2}=\dot{U}_{2} \text { and } \dot{T}_{01}=0, \quad \text { for } \quad x_{2}=R_{c}+U_{2}
\end{aligned}
$$

where $\dot{U}_{1}$ and $\dot{U}_{2}$ are determined such that a constant ratio of the average true stresses is maintained

$$
\frac{\sigma_{2}}{\sigma_{1}}=\rho
$$

Defining the triaxiality ratio, $T$, as the ratio of the macroscopic effective stress to the macroscopic hydrostatic stress gives the following relation (Koplik and Needleman, 1988)

$$
T=\frac{1+2 \rho}{3(1-\rho)}
$$

In addition to the conventional boundary conditions, $\dot{t}_{0}=0$ is specified along the entire surface of the material modeled. At the surface of the cell this constitutes the appropriate symmetry boundary condition, while at the void surface it models that there is no constraint on plastic flow at the free surface. Also, for internal elastic-plastic boundaries a vanishing constraint on plastic flow is imposed $\left(\dot{t}_{0}=0\right)$. Here, the choice is not trivial, but as argued in

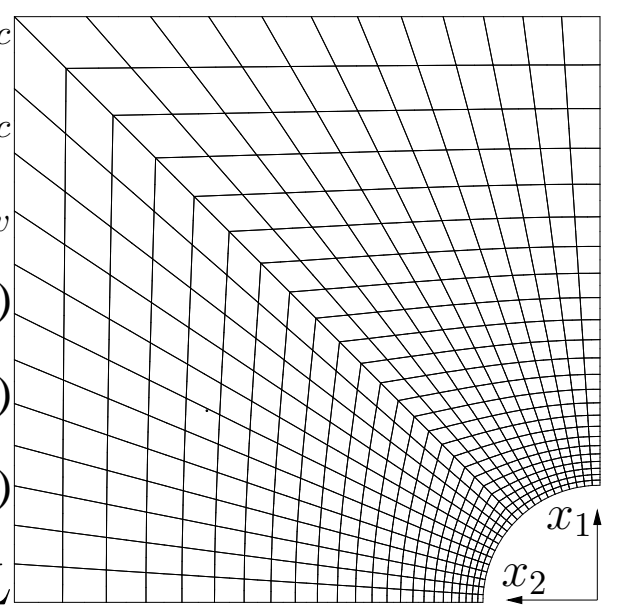

Figure 2: A typical example of a fi nite element mesh used for the numerical computations. 
Niordson and Hutchinson (2003), this models a material where dislocations are free to penetrate the current elastic-plastic boundary.

The nonlocal serendipity elements developed in Niordson and Tvergaard (2005) are used to model the power-law hardening material behavior. Further details on the numerical implementation are found in Niordson and Hutchinson (2003), Niordson and Redanz (2004), and Niordson and Tvergaard (2005, 2006).

Figure 2 shows a typical finite element mesh used for the numerical analyses.

\section{RESULTS}

The true stress as a function of the logarithmic strain is shown in Figure 3 a for conventional materials with different void volume fractions and for different loading ratios, $\rho$. The largest value of $\rho=0.75$ corresponds to the triaxiality ratio $T \approx 3.3$, which is roughly the triaxiality in in the vicinity of a crack tip for lightly hardening solids (Koplik and Needleman, 1988). Figure $3 \mathrm{~b}$ shows the relative void growth as a function of strain. For $R_{v}=0$ there are no voids so the loading is homogeneous. For $R_{v} / R_{c}=0.05$ and $R_{v} / R_{c}=0.10$ the void volume fractions are $f \approx 8.33 \cdot 10^{-5}$ and $f \approx 6.67 \cdot 10^{-4}$, respectively, while for the largest void size compared to

(a)

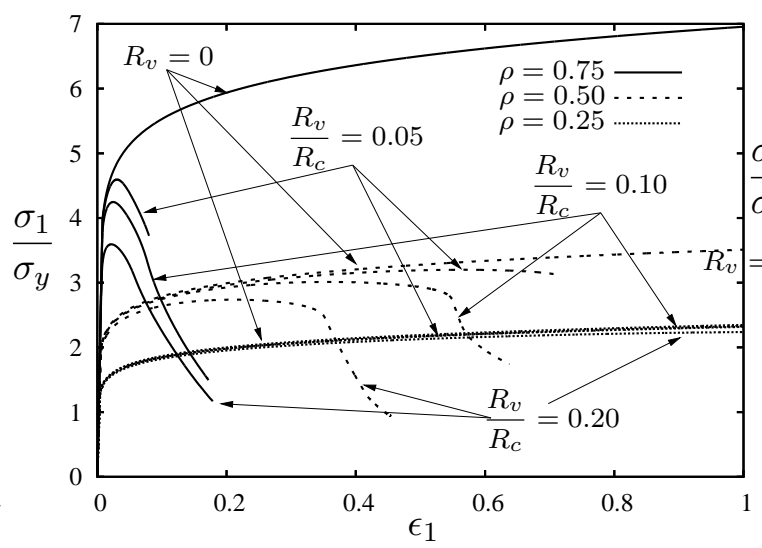

(b)

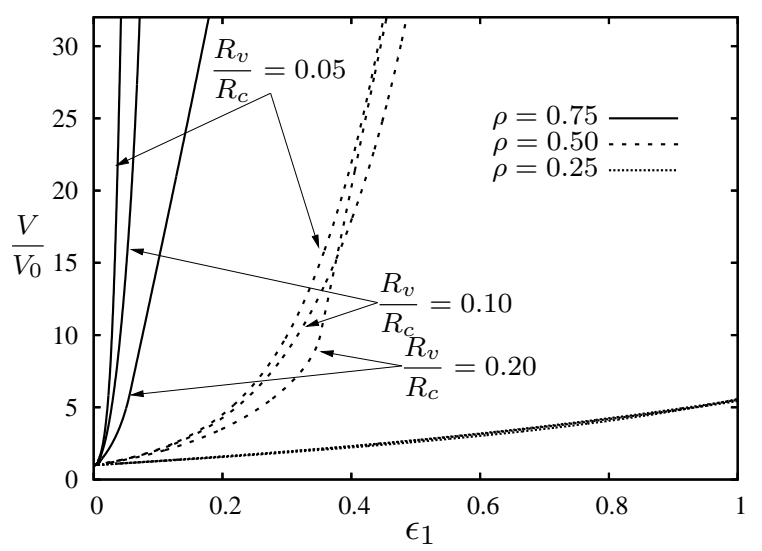

Figure 3: Conventional results $\left(l_{*}=0\right)$ for a material without voids and for materials with three different void volume fractions, where the void distribution is such that there is an equal in-plane and out-of-plane spacing $\left(L_{c} / R_{c}=1\right)$. The analyses are carried out for three different values of the ratio of transverse stress to axial stress, $\rho$. The material parameters are given by $\sigma_{y} / E=0.004, \nu=1 / 3$ and $n=10$. (a) Shows the overall response in terms of the true stress as a function of logarithmic strain, and (b) shows the relative void growth as a function of strain. 
(a)

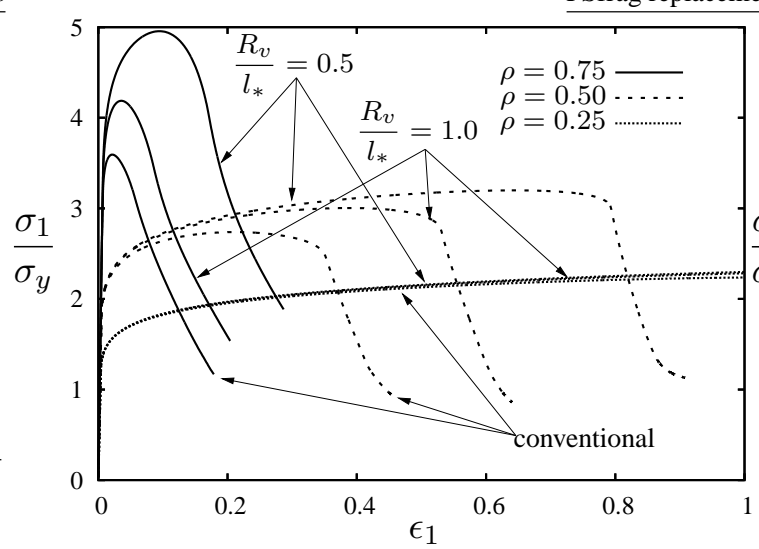

(b)

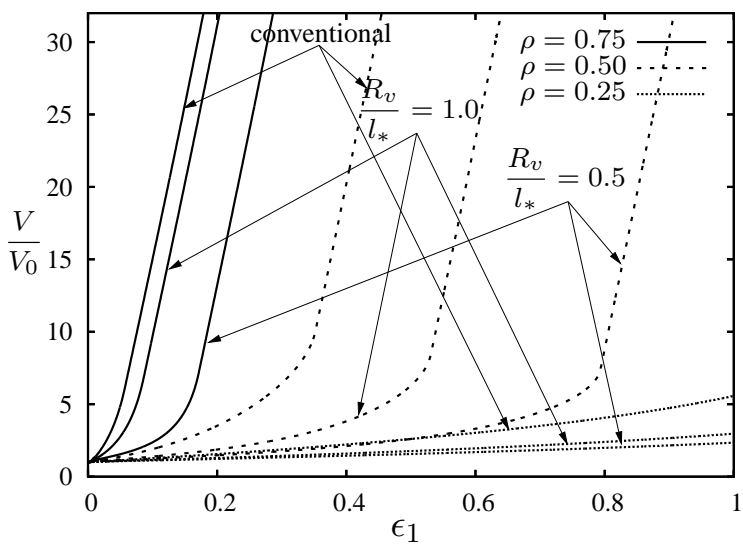

Figure 4: Size dependent results for a material with a large void volume fraction $f \approx 5.33 \cdot 10^{-3}$ (corresponding to $\left.R_{v} / R_{c}=0.2\right)$ with equal in-plane and out-of-plane spacing $\left(L_{c} / R_{c}=1\right)$. Both conventional and gradient dependent results with $R_{v} / l_{*}=1.0$ and $R_{v} / l_{*}=0.5$ are shown for three different stress ratios. The conventional material parameters are given by $\sigma_{y} / E=0.004, \nu=1 / 3$ and $n=10$. (a) Shows the overall response in terms of the true stress as a function of strain, and (b) shows the relative void growth.

the size of the cell $\left(R_{v} / R_{c}=0.2\right)$ the void volume fraction is $f \approx 5.33 \cdot 10^{-3}$. For the two larger values of the stress ratio, $\rho$, it is seen that introducing voids in the material leads to overall softening of the material during void growth. This is due to void growth toward coalescence (Koplik and Needleman, 1988). For $\rho=0.75$ the strain level at which the softening begins is significantly smaller than for $\rho=0.50(T \approx 1.3)$, due to much more dramatic void growth at low strains. At large deformation levels the finite element mesh becomes distorted. Hence, the analyses are stopped when the relative void growth reaches 200, except for the analyses with the initial void size of $R_{v} / R_{c}=0.20$, which are stopped at $\Delta V / V_{0}=32$.

When the void size is on the micron to sub-micron scale it is expected that geometrically necessary dislocations due to large strain gradients (Ashby, 1970) will lead to additional hardening over macroscopic voids with the same void volume fraction even though the overall loading is homogeneous. This is reported in a number of investigations for hydrostatic loading by Fleck and Hutchinson (1997), Huang et al. (2000) and Fleck and Hutchinson (2001), and for axisymmetric loading conditions by Liu et al. (2003, 2005), Wen et al. (2005), Tvergaard and Niordson (2004) and Niordson and Tvergaard (2006, 2007). Similar size effects are observed in metal matrix composites (Lloyd, 1994) which are well captured by gradient theories of plasticity (Bittencourt et al., 2003; Niordson, 2003). 
In Figure 4 results corresponding to those in Figure 3 are shown for the larger void volume fraction $\left(f \approx 5.33 \cdot 10^{-3}\right.$ ) specified by $R_{v} / R_{c}=0.2$ for different void sizes. Assuming a fixed material length parameter, $l_{*}$, which is on the micron scale, the ratio $R_{v} / l_{*}$ specifies the void size. It is observed that decreasing the void size leads to a significant increase in the attainable stress levels, especially for $\rho=0.75$. This is due to the suppression of void growth as a result of gradient hardening, which moreover has the effect that the maximum value of the overall stressstrain curve occurs at a lager strain. For $\rho=0.5$ a sharp kink in the response curves is observed, which accompanies significant void growth. This is closely related to the onset of what Koplik and Needleman (1988) refer to as the uniaxial straining deformation mode which can be used to define the onset of void coalescence. Figure 4 shows that the this onset of void coalescence is significantly delayed for smaller voids, and that the strain at the onset of coalescence is significantly increased as the voids become comparable in size to the material length parameter. However, the variation of the maximum stress is much smaller than for $\rho=0.75$. At low stress triaxialities ( $\rho=0.25$ corresponding to $T \approx 0.67$ ) the overall response is almost unaffected by void size (specified by the ratio $R_{v} / l_{*}$ ), since void growth is rather limited. The amount of void growth is quite limited for this low triaxiality, and relatively independent of size at small strains. On the other hand for larger deformation levels, the void size effect increases. These

(a)

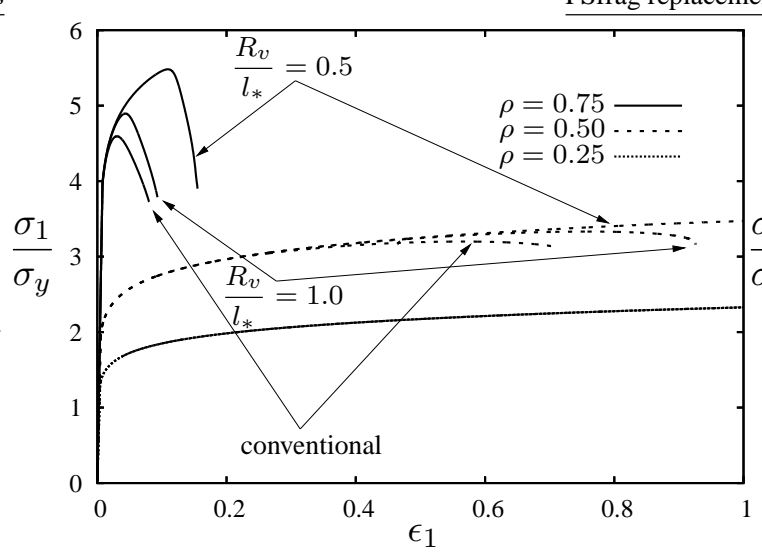

(b)

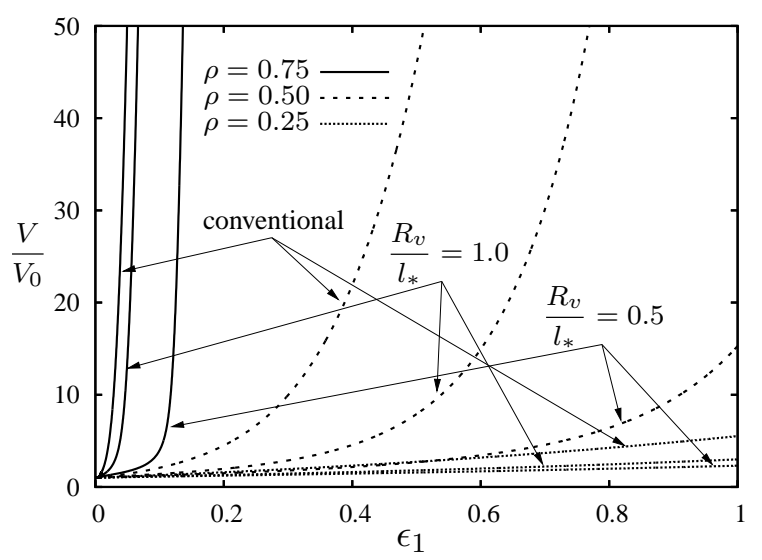

Figure 5: Size dependent results for a material with a small void volume fraction $f \approx 8.33 \cdot 10^{-5}$ (corresponding to $R_{v} / R_{c}=0.05$ ). Both conventional and gradient dependent results with $R_{v} / l_{*}=1.0$ and $R_{v} / l_{*}=0.5$ are shown for three different stress ratios. The conventional material parameters are given by $\sigma_{y} / E=0.004, \nu=1 / 3$ and $n=10$. (a) Shows the overall response in terms of the true stress as a function of strain, and (b) shows the relative void growth. 
findings are in qualitative agreement with Liu et al. (2003) who found large effects of void size for large mean stresses, while the size effect in void growth was found to be insignificant for small values of the mean stress, though it was found to be amplified at large remote deformation levels.

Results for a smaller void volume fraction with $R_{v} / R_{c}=0.05\left(f \approx 8.33 \cdot 10^{-5}\right)$ are presented in Figure 5. Also here, it is seen that gradient effects lead to delayed void growth and an increased maximum stress when voids become smaller, as is most clearly observed for $\rho=0.50$ and $\rho=0.75$. For the higher stress ratio $(\rho=0.75)$ a significant increase in the stress level with decreasing void size is observed. This is followed by an increasingly steep stress decay with decreasing initial void size as the voids show dramatic growth during coalescence. For the same strain level the amount of relative void growth can easily vary an order of magnitude or more in materials with initial void sizes that are just a factor of two different.

For $\rho=0.75$ response curves and curves of relative void growth are shown in Figure 6 for different initial void volume fractions. The initial void volume fraction ranges from $6.67 \cdot 10^{-7}$ $\left(R_{v} / R_{c}=0.01\right)$ to $5.33 \cdot 10^{-3}\left(R_{v} / R_{c}=0.20\right)$. The response curve for a material without voids is also shown in Figure 6a. For the conventional material it is seen that decreasing the void volume fraction leads to an increase in the overall strength of the material, even though the relative growth of the voids increases significantly at any given level of overall strain. The

$(a)$

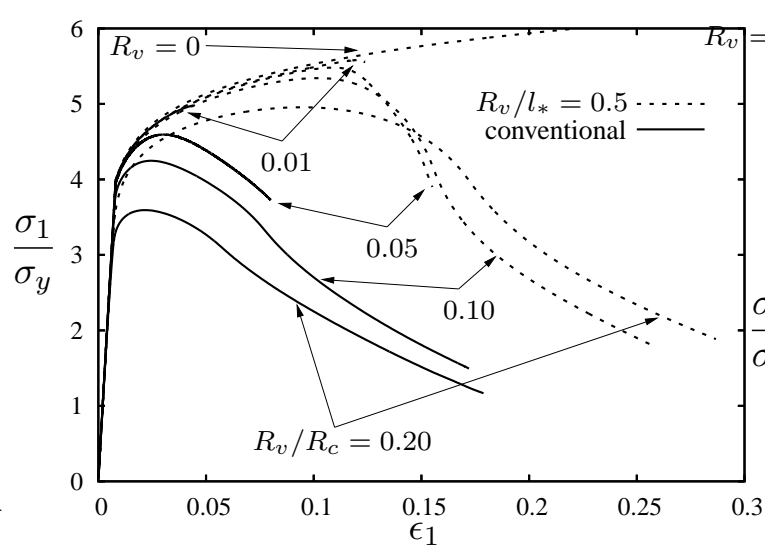

(b)

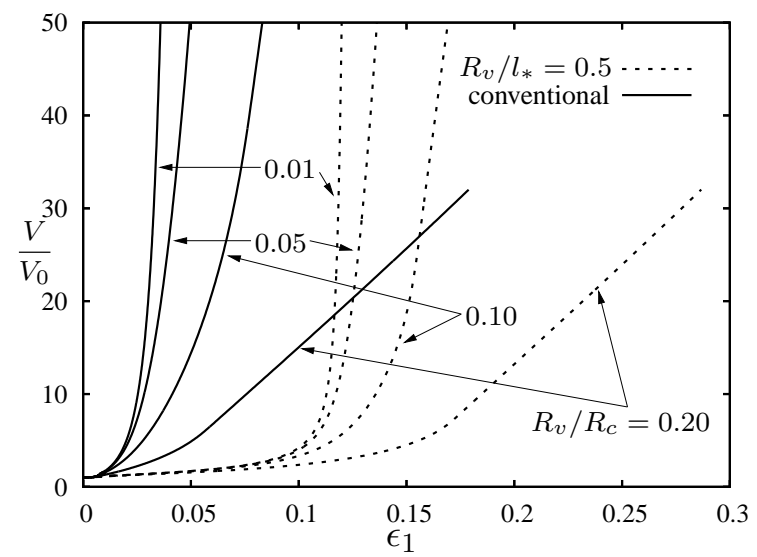

Figure 6: Results for initially large voids (conventional) compared to micron scale voids $\left(R_{v} / l_{*}=0.5\right)$ for different void volume fractions (specifi ed by the value of $R_{v} / R_{c}$ ). The stress ratio is $\rho=0.75$. The conventional material parameters are given by $\sigma_{y} / E=0.004, \nu=1 / 3$ and $n=10$. (a) Shows the overall response in terms of the true stress as a function of strain, and (b) shows the relative void growth. 
(a)

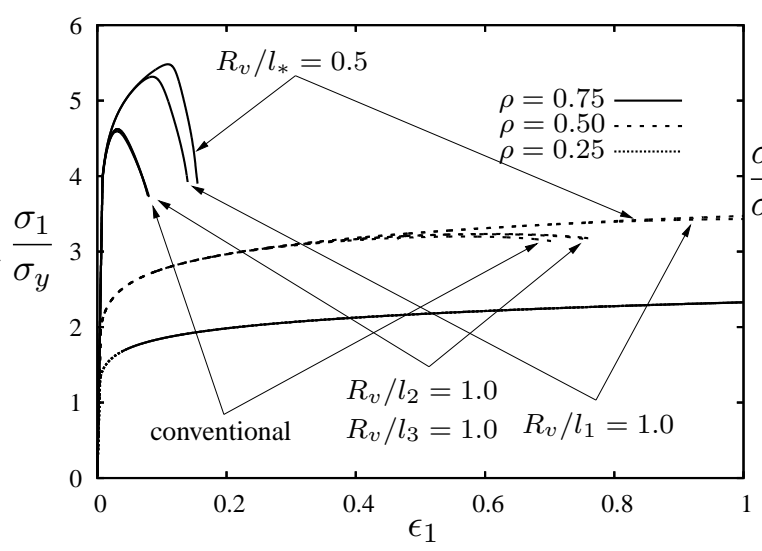

(b)

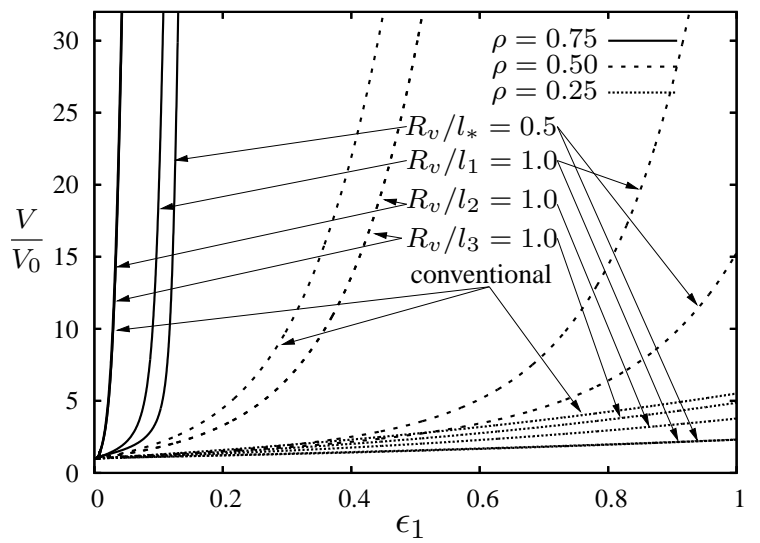

Figure 7: Results for the multi-parameter model compared to the single parameter model for a void volume fraction $f \approx 8.33 \cdot 10^{-5}\left(R_{v} / R_{c}=0.05\right)$. For the multi-parameter model each of the length parameters are set equal to $R_{v}$ one by one keeping the others zero. The results are presented for three different stress-triaxialities. (a) Shows the overall response in terms of the true stress as a function of strain, and (b) shows the relative void growth.

strain level at which the maximum stress level is attained increases with decreasing void volume fraction. When the voids are on the micron scale $\left(R_{v} / l_{*}=0.5\right)$, the attainable stress levels are increased due to significant suppression of void growth at small deformation levels.

Studies of the growth of a single void under hydrostatic loading $(\rho=1)$ (Fleck and Hutchinson, 2001) shows that $l_{2}$ has no influence in the multi-parameter theory (Equation (1)). Furthermore, they report that $l_{3}$ only has a minor influence, and that $l_{1}$ is the important length parameter for void growth under hydrostatic loading conditions. For $\rho=0.9$, it was confirmed by Niordson and Tvergaard (2006), in their studies of cavitation instabilities, that $l_{2}$ and $l_{3}$ have an insignificant influence on growth of sparsely distributed voids. However, at lower values of the stress ratio $\rho$, it must be expected that both $l_{2}$ and $l_{3}$ influence void growth. For $R_{v} / R_{c}=0.05\left(f \approx 8.33 \cdot 10^{-5}\right)$ Figure 7 shows response curves and curves of relative void growth for different stress ratios and different length parameters. The figure shows that when $\rho=0.75$ the length parameters $l_{2}$ and $l_{3}$ has an insignificant influence on both the overall response (Figure 7a) and the amount of void growth (Figure 7b). On the other hand, void growth is delayed somewhat for $\rho=0.50$, when setting the length parameters $l_{2}$ and $l_{3}$ equal to the initial void radius one by one. This leads to response curves that are affected slightly toward higher overall ductility. For $\rho=0.25$ the amount of void growth is very small so even though gradient effects (through either of the length parameters) does affect the amount of relative void 
(a)

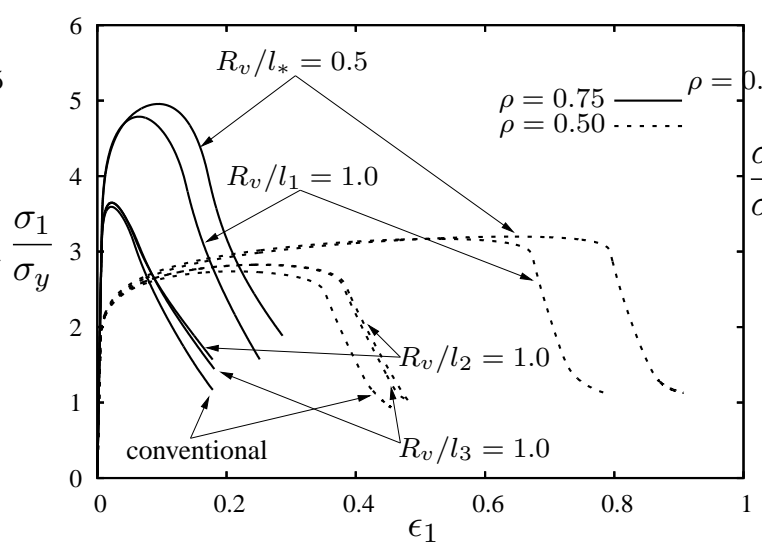

(b)

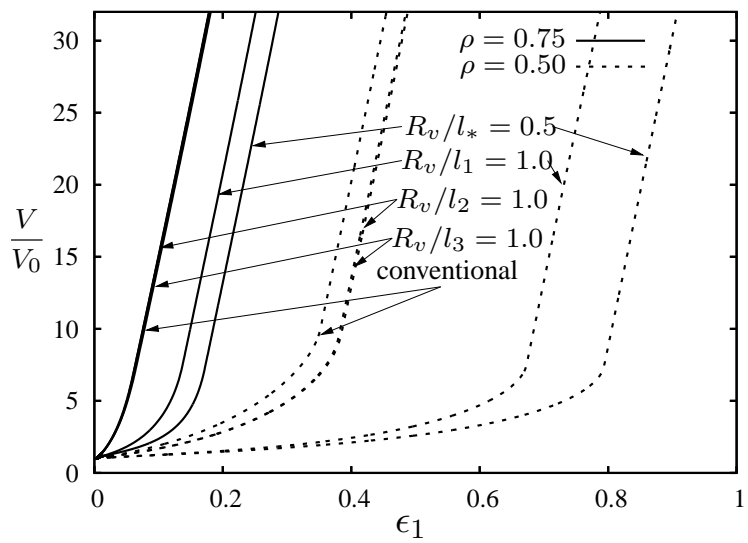

Figure 8: For a large void volume fraction $f \approx 5.33 \cdot 10^{-3}\left(R_{v} / R_{c}=0.2\right)$ this fi gure shows results for the multiparameter model compared to the single parameter model for different stress-ratios. For the multi-parameter model each of the length parameters are set equal to $R_{v}$ one by one keeping the others zero. The results are presented for three different stress-triaxialities. (a) Shows the overall response in terms of the true stress as a function of strain, and (b) shows the relative void growth.

growth, the total void growth is so small that it has no effect on the overall response within the deformation levels studied here.

For all the values of $\rho$ studied in Figure 7 it is seen that the important length parameter in relation to void growth is $l_{1}$. The figure also confirms for $\rho<1$, what Fleck and Hutchinson (2001) showed for spherically symmetric void expansion, i.e. that gradient effects predicted by the single parameter version Equation (2) are quantitatively similar to those predicted by the multi-parameter version when $l_{*}$ is about twice the value of $l_{1}$.

At larger void volume fractions the interaction between the voids changes the stress state such that the dominant role of the length parameter $l_{1}$ could be challenged by $l_{2}$ and $l_{3}$. However, Figure 8 shows that the influence of these two length parameters is still very limited, as compared to that of $l_{1}$. Figure 8 shows that even though $l_{2}$ and $l_{3}$ have almost no influence on the amount of void growth as a function of strain for $\rho=0.5$, they do have a minor influence on the response curves. This effect is similar to the effect of these parameters on plane strain sheet necking (see Niordson and Redanz (2004) and Niordson and Tvergaard, 2005), as $l_{2}$ and $l_{3}$ tend to limit the development of the neck in the ligaments along the cell sides during coalescence. For the higher stress ratio, $\rho=0.75, l_{2}$ and $l_{3}$ have a moderate influence on both response curves and the amount of relative void growth. However, also for the large voids it is 
seen that the important length parameter in the multi-parameter model is $l_{1}$, and also here the results show that a similar effect on void growth is obtained in the single-parameter model with $l_{*}$ being twice as large as $l_{1}$.

\section{DISCUSSION}

It is shown how a constitutive length parameter on the micron scale changes void growth quantitatively for micron size voids. When voids become smaller and comparable to the internal material length parameter, void growth is significantly suppressed consistent with findings of Fleck and Hutchinson (1997, 2001), Huang et al. (2000), and Liu et al. (2003, 2005). This has the effect of increasing the attainable stress level and the strain at the onset of void coalescence.

At relatively large void volume fraction $\left(f \approx 5.33 \cdot 10^{-3}\right)$ Figure 4 shows that especially for large triaxialities $(T \approx 3.3)$ the load carrying capacity of the material is significantly increased for micron scale voids. For a smaller stress triaxiality of $T \approx 1.3$ this capacity is still increased for small voids but just moderately, while the ductility of the material still increases significantly due to the delay of void coalescence.

For smaller initial void volume fractions $f \approx 8.33 \cdot 10^{-5}$ Figure 5 shows that the initial void size has an important effect on the overall response when the stress triaxiality is large. At more moderate stress triaxialities the effect of void size on the overall response is much smaller consistent with the findings of Liu et al. (2003). However, it must be expected that the ductility of the material is much influenced, as a reduced void size, relative to the material length, still leads to a significant suppression of void growth at large levels of deformation.

When void sizes are on the micron scale, the effect of varying the initial void volume fraction, is studied in Figure 6 for a high stress triaxiality $(\rho=0.75)$. It is found that increasing the void volume fraction for micron size voids leads to smaller peak levels in the stress, which is also the case for large voids (conventional results). The results show that the ductility of the material increases with increasing initial void volume fraction. However, a comparison of the results in the Figures 4 and 5 shows that for a lower stress triaxialities $(\rho=0.5)$, the increase in void volume fraction leads to decreasing ductility for both macroscopic and micron scale voids.

The present studies of the multi length parameter model by Fleck and Hutchinson (2001) confirms earlier studies (Fleck and Hutchinson, 2001; Niordson and Tvergaard, 2006) that the important length parameter is $l_{1}$, which is associated with stretch gradients. However, for large void volume fractions or moderate to low stress triaxialities also the length parameters $l_{2}$ and 
(a)

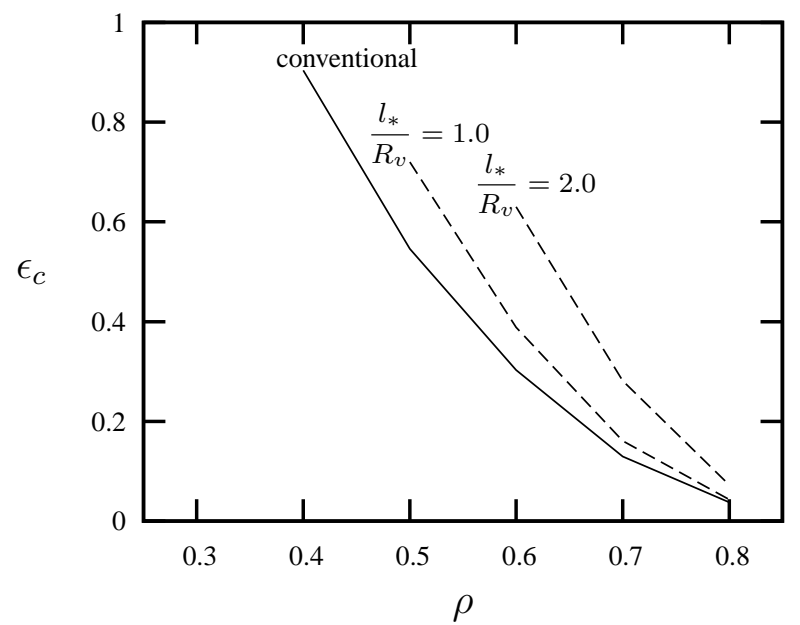

(b)

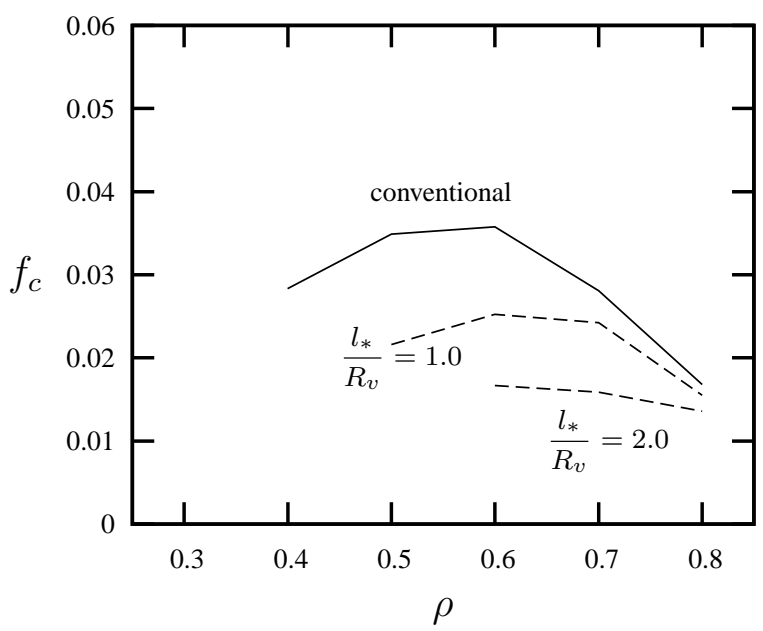

Figure 9: For an initial void volume fraction of $f_{0} \approx 6.67 \cdot 10^{-4}$, the fi gure shows (a) the strain and (b) the void volume fraction at the onset of coalescence, both as a function of the stress ratio, and for different initial void sizes.

$l_{3}$ have some effect in suppressing void growth and the final deformation in the ligaments in the stage of void coalescence. Furthermore, it was confirmed for a wide spectrum of stress triaxialities and a large span of initial void volume fractions that $l_{1}$ has a similar effect on void growth as $l_{*}$, when chosen half as large.

Defining the onset of void coalescence as the onset of the uniaxial straining mode, Figure 9(a) shows the strain at the onset of coalescence as a function of the stress ratio, $\rho$, for different void sizes, when the initial void volume fraction is $f_{0} \approx 6.67 \cdot 10^{-4}$. It is seen that the strain at the onset of localization decreases with increasing stress ratio for all the void sizes considered. Furthermore, the figure shows that the ductility increases with decreasing void size. Figure 9(b) shows the void volume fraction at the onset of void coalescence as a function of $\rho$. It can be observed that the void volume fraction decreases with decreasing void size at the onset of coalescence.

For an initial void volume fraction of $f_{0} \approx 5.33 \cdot 10^{-3}$ similar results are shown in Figure 10(a) and (b). It is observed that also for this initial void volume fraction the strain at the onset of localization decreases with increasing stress ratio for all values of the initial void size. Also here, the ductility is observed to increase with decreasing void size. On the other hand, the void volume fraction at the onset of coalescence in Figure 10(b) only decreases with decreasing void size for small values of $\rho$, whereas it increases with decreasing void size for large triaxialities. 
(a)

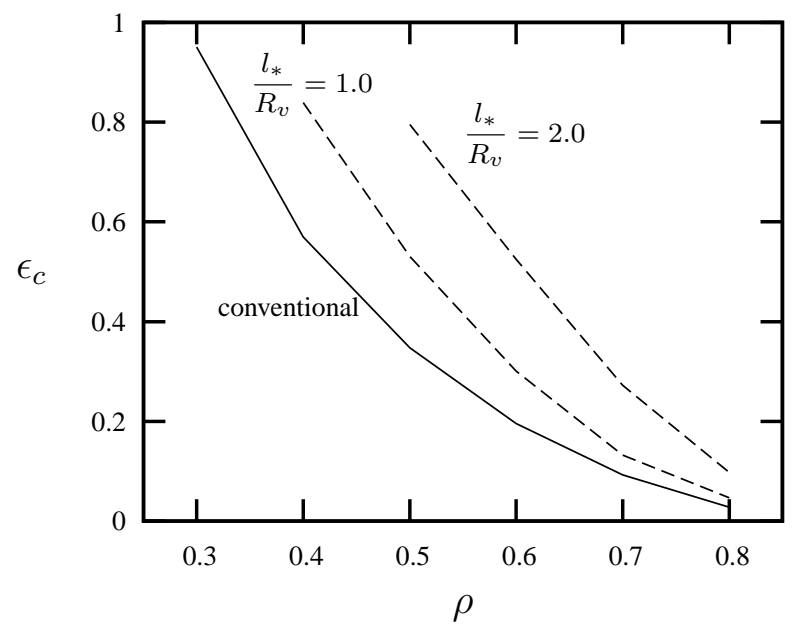

(b)

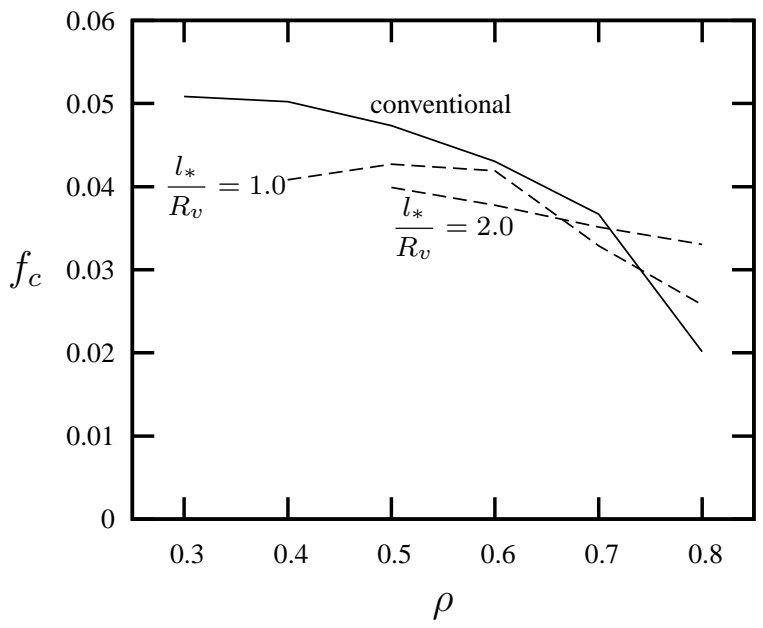

Figure 10: For an initial void volume fraction of $f_{0} \approx 5.33 \cdot 10^{-3}$, the fi gure shows (a) the strain and (b) the void volume fraction at the onset of coalescence, both as a function of the stress ratio, and for different initial void sizes.

Consistent with the findings for conventional materials reported in Tvergaard (1990), the void volume fraction at the onset of coalescence is observed to increase with increasing initial void volume fraction, also for gradient dependent materials with micron scale voids. It must be concluded that there is a significant influence of the stress ratio and initial void volume fraction as well as void size on the critical porosity at the onset of coalescence.

In the results presented, the material hardening is specified by a power-law relation with $n=10$. Simulations with higher $(n=5)$ as well as lower material hardening $(n=20)$ have also been carried out, and it is found that the effect of size increases with material hardening. This is consistent with findings for cavitation instabilities (Huang et al., 2000; Niordson and Tvergaard, 2006).

In conclusion, the present studies show that the suppression of void growth on the micron scale leads to larger attainable stress levels when compared to predictions of conventional theory, as well as a delay in the onset of localization which gives rise to a more ductile material behavior.

\section{ACKNOWLEDGMENTS}

This work is financially supported by the Danish Technical Research Council in a project entitled Modeling Plasticity at the Micron Scale. 


\section{REFERENCES}

Acharya, A. and Bassani, J. L. (1996), On non-local flow theories that preserve the classical structure of incremental boundary value problems, in A. Pineau and A. Zaoui, eds, 'IUTAM Symposium on Micromechanics of Plasticity and Damage of Multiphase Materials', Kluwer Academic Publishers, pp. 3-9.

Aifantis, E. C. (1984), 'On the microstructural origin of certain inelastic models', Transactions of the ASME. Journal of Engineering Materials and Technology 106(4), 326-330.

Ashby, M. (1970), ‘The deformation of plastically non-homogeneous materials', Philosophical Magazine 21, 399-424.

Bittencourt, E., Needleman, A., Gurtin, M. and Van der Giessen, E. (2003), 'The deformation of plastically non-homogeneous materials', Journal of the Mechanics and Physics of Solids 51, 281-310.

de Borst, R. and Mühlhaus, H.-B. (1992), 'Gradient-dependent plasticity: formulation and algorithmic aspects', International Journal for Numerical Methods in Engineering 35, 521539.

de Borst, R. and Pamin, J. (1996), 'Some novel developments in finite element procedures for gradient-dependent plasticity', International Journal for Numerical Methods in Engineering 39, 2477-2505.

Fleck, N. A. and Hutchinson, J. W. (1997), Strain gradient plasticity, in J. W. Hutchinson and T. Y. Wu, eds, 'Advances in Applied Mechanics', Vol. 33, Academic Press, pp. 295-361.

Fleck, N. A. and Hutchinson, J. W. (2001), ‘A reformulation of strain gradient plasticity’, Journal of the Mechanics and Physics of Solids 49, 2245-2271.

Fleck, N. A., Muller, G. M., Ashby, M. F. and Hutchinson, J. W. (1994), 'Strain gradient plasticity: Theory and experiment', Acta Metallurgica et Materialia 42(2), 475-487.

Gao, H., Huang, Y., Nix, W. and Hutchinson, J. W. (1999), 'Mechanism-based strain gradient plasticity-I. analysis', Journal of the Mechanics and Physics of Solids 47, 1239-1263. 
Gologanu, M., Leblond, J.-B. and Devaux, J. (1993), 'Approximate models for ductile metals containing non-spherical voids - case of axisymmetric prolate ellipsoidal cavities', Journal of the Mechanics and Physics of Solids 41, 1723-1754.

Gologanu, M., Leblond, J.-B. and Devaux, J. (1994), 'Approximate models for ductile metals containing non-spherical voids - case of axisymmetric oblate ellipsoidal cavities', Journal of Engineering Materials and Technology 116, 290-297.

Gudmundson, P. (2004), 'A unified treatment of strain gradient plasticity', Journal of the Mechanics and Physics of Solids 52, 1379-1406.

Gurson, A. (1977), 'Continuum theory of ductile rupture by void nucleation and growth: Part I. Yield criteria and flow rules for porous ductile media', Journal of Engineering Materials and Technology 99, 2-15.

Gurtin, M. E. (2002), 'A gradient theory of single-crystal viscoplasticity that accounts for geometrically necessary dislocations', Journal of the Mechanics and Physics of Solids 50, 532.

Haque, M. A. and Saif, M. T. A. (2003), 'Strain gradient effect in nanoscale thin films', Acta Materialia 51(11), 3053-3061.

Huang, Y., Gao, H., Nix, W. and Hutchinson, J. W. (2000), 'Mechanism-based strain gradient plasticity-II. analysis', Journal of the Mechanics and Physics of Solids 48, 99-128.

Huang, Y., Qu, S., Hwang, K. C., Li, M. and Gao, H. (2004), 'A conventional theory of mechanism-based strain gradient plasticity', International Journal of Plasticity 20, 753 782.

Johnson, K. (1970), 'The correlation of indentation experiments', Journal of the Mechanics and Physics of Solids 18, 115-126.

Koplik, J. and Needleman, A. (1988), 'Void growth and coalescence in porous plastic solids', International Journal of Solids and Structures 24, 835-853.

Kysar, J. W., Gan, Y. X. and Mendez-Arzuza, G. (2005), 'Cylindrical void in a rigid-ideally plastic single crystal. Part I: Anisotropic slip line theory solution for face-centered cubic crystals', International Journal of Plasticity 21, 1481-1520. 
Leblond, J.-B., Perrin, G. and Devaux, J. (1994), 'Bifurcation effects in ductile metals with damage delocalization', Journal of Applied Mechanics 61, 236-242.

Liu, B., Huang, Y., Li, M., Hwang, K. C. and Liu, C. (2005), 'A study of the void size effect based on the Taylor dislocation model', International Journal of Plasticity 21, 2107-2122.

Liu, B., Qiu, X., Huang, Y., Hwang, K. C., Li, M. and Liu, C. (2003), 'The size effect on void growth in ductile materials', Journal of the Mechanics and Physics of Solids 51, 11711187.

Lloyd, D. J. (1994), 'Particle reinforced aluminium and magnesium matrix composites', International Materials Review 39(1), 1-23.

Ma, Q. and Clarke, D. R. (1995), 'Size dependent hardness of silver single crystals', Journal of Materials Research 10, 853-863.

Marsh, D. (1964), 'Plastic flow in glass', Proceedings of the Royal Society of London. Series A 279, 420-435.

McElwain, D., Roberts, A. and Wilkins, A. (2006), 'Yield criterion of porous materials subjected to complex stress states', Acta Materialia 54, 1995-2002.

Needleman, A. (1972), 'Void growth in and elastic-plastic medium', Journal of Applied Mechanics 39, 974-970.

Niordson, C. F. (2003), 'Strain gradient plasticity effects in whisker-reinforced metals', Journal of the Mechanics and Physics of Solids 51, 1863-1883.

Niordson, C. F. and Hutchinson, J. W. (2003), 'Non-uniform plastic deformation of micron scale objects', International Journal for Numerical Methods in Engineering 56, 961-975.

Niordson, C. F. and Redanz, P. (2004), 'Size-effects in plane strain sheet-necking', Journal of the Mechanics and Physics of Solids 52, 2431-2454.

Niordson, C. F. and Tvergaard, V. (2005), 'Instabilities in power law gradient hardening materials', International Journal of Solids and Structures 42, 2559-2573.

Niordson, C. F. and Tvergaard, V. (2006), 'Size-effects on cavitation instabilities', Journal of Applied Mechanics 73, 246-253. 
Niordson, C. F. and Tvergaard, V. (2007), 'Size-effects in porous metals', To appear in 'Modelling and Simulation in Materials Science and Engineering'.

Pardoen, T. and Hutchinson, J. W. (2000), 'An extended model for void growth and coalescence', Journal of the Mechanics and Physics of Solids 48, 2467-2512.

Rice, J. and Tracey, D. (1969), 'On the ductile enlargement of voids in triaxial stress fields', Journal of the Mechanics and Physics of Solids 17, 201-217.

Stelmashenko, N. A., Walls, M. G., Brown, L. M. and Milman, Y. V. (1993), 'Microindentations on W and Mo oriented single crystals: An SEM study', Acta Metallurgica et Materialia 41(10), 2855-2865.

Stölken, J. S. and Evans, A. (1998), 'A microbend test method for measuring the plasticity length scale', Acta Materialia 46(14), 5109-5115.

Swadener, J., George, E. and Pharr, G. (2002), 'The correlation of the indentation size effect measured with indenters of various shapes', Journal of the Mechanics and Physics of Solids 50, 681-694.

Tvergaard, V. (1982), 'On localization in ductile materials containing spherical voids', International Journal of Fracture 18(4), 237-252.

Tvergaard, V. (1990), Material failure by void growth to coalescence, in J. W. Hutchinson and T. Y. Wu, eds, 'Advances in Applied Mechanics', Vol. 27, Academic Press, pp. 83-151.

Tvergaard, V. and Needleman, A. (1997), 'Nonlocal effects on localization in a void-sheet', International Journal of Solids and Structures 34, 2221-2238.

Tvergaard, V. and Niordson, C. F. (2004), 'Nonlocal plasticity effects on interaction of different size voids', International Journal of Plasticity 20, 107-120.

Wei, Y. and Hutchinson, J. W. (2003), 'Hardness trends in micron scale indentation', Journal of the Mechanics and Physics of Solids 51, 2037-2056.

Wen, J., Huang, Y., Hwang, K. C., Liu, C. and Li, M. (2005), 'The modified Gurson model accounting for the void size effect', International Journal of Plasticity 21, 381-395. 


\section{FIGURE CAPTIONS}

Figure 1. Cell model for a material with an array of voids. (a) Hexagonal distribution of voids with a cylindrical cell indicated by the circular dashed line. (b) A part of a layer of voids. (c) Using the symmetry of the problem half a void can be modeled in an axi-symmetric cell. The cell radius and length are denoted $R_{c}$ and $L_{c}$, respectively, and the void radius is denoted $R_{v}$.

Figure 2. A typical example of a finite element mesh used for the numerical computations.

Figure 3. Conventional results $\left(l_{*}=0\right)$ for a material without voids and for materials with three different void volume fractions, where the void distribution is such that there is an equal in-plane and out-of-plane spacing $\left(L_{c} / R_{c}=1\right)$. The analyses are carried out for three different values of the ratio of transverse stress to axial stress, $\rho$. The material parameters are given by $\sigma_{y} / E=0.004, \nu=1 / 3$ and $n=10$. (a) Shows the overall response in terms of the true stress as a function of logarithmic strain, and (b) shows the relative void growth as a function of strain.

Figure 4. Size dependent results for a material with a large void volume fraction $f \approx 5.33 \cdot 10^{-3}$ (corresponding to $\left.R_{v} / R_{c}=0.2\right)$ with equal in-plane and out-of-plane spacing $\left(L_{c} / R_{c}=1\right)$. Both conventional and gradient dependent results with $R_{v} / l_{*}=1.0$ and $R_{v} / l_{*}=0.5$ are shown for three different stress ratios. The conventional material parameters are given by $\sigma_{y} / E=$ 0.004, $\nu=1 / 3$ and $n=10$. (a) Shows the overall response in terms of the true stress as a function of strain, and (b) shows the relative void growth.

Figure 5. Size dependent results for a material with a small void volume fraction $f \approx 8.33 \cdot 10^{-5}$ (corresponding to $R_{v} / R_{c}=0.05$ ). Both conventional and gradient dependent results with $R_{v} / l_{*}=1.0$ and $R_{v} / l_{*}=0.5$ are shown for three different stress ratios. The conventional material parameters are given by $\sigma_{y} / E=0.004, \nu=1 / 3$ and $n=10$. (a) Shows the overall response in terms of the true stress as a function of strain, and (b) shows the relative void growth. Figure 6. Results for initially large voids (conventional) compared to micron scale voids $\left(R_{v} / l_{*}=\right.$ 0.5 ) for different void volume fractions (specified by the value of $R_{v} / R_{c}$ ). The stress ratio is $\rho=0.75$. The conventional material parameters are given by $\sigma_{y} / E=0.004, \nu=1 / 3$ and $n=10$. (a) Shows the overall response in terms of the true stress as a function of strain, and (b) shows the relative void growth.

Figure 7. Results for the multi-parameter model compared to the single parameter model for a void volume fraction $f \approx 8.33 \cdot 10^{-5}\left(R_{v} / R_{c}=0.05\right)$. For the multi-parameter model each of 
the length parameters are set equal to $R_{v}$ one by one keeping the others zero. The results are presented for three different stress-triaxialities. (a) Shows the overall response in terms of the true stress as a function of strain, and (b) shows the relative void growth.

Figure 8. For a large void volume fraction $f \approx 5.33 \cdot 10^{-3}\left(R_{v} / R_{c}=0.2\right)$ this figure shows results for the multi-parameter model compared to the single parameter model for different stress-ratios. For the multi-parameter model each of the length parameters are set equal to $R_{v}$ one by one keeping the others zero. The results are presented for three different stresstriaxialities. (a) Shows the overall response in terms of the true stress as a function of strain, and (b) shows the relative void growth.

Figure 9. For an initial void volume fraction of $f_{0} \approx 6.67 \cdot 10^{-4}$, the figure shows (a) the strain and (b) the void volume fraction at the onset of coalescence, both as a function of the stress ratio, and for different initial void sizes.

Figure 10. For an initial void volume fraction of $f_{0} \approx 5.33 \cdot 10^{-3}$, the figure shows (a) the strain and (b) the void volume fraction at the onset of coalescence, both as a function of the stress ratio, and for different initial void sizes. 